\title{
Collection, Identification, Phytochemical analysis and Phyto toxicity test of Wood inhabiting Fungi Ganoderma lucidum (Curt.Fr.)P.Karst.
}

\author{
M.Nithya* V.Ambikapathy and A.Panneerselvam. \\ P.G and Research Department of Botany and Microbiology, A.V.V.M Sri Pushpam College (Autonomous),
} Poondi-613 503, Thanjavur, Tamilnadu, India.

Article history: Received: 18 November 2013, revised: 10 December 2013, accepted: 11January 2014, Available online: 3 April 2014

\begin{abstract}
Plan: In this study 10 strains of mushroom Ganoderma lucidum were collected from various places and decided to screen their phytotoxicity activity and phytochemical constitution.

Preface: The popular mushroom Ganoderma lucidum (Reishi) was a bracket fungus and has been widely used for the promotion of health and longevity in Asian countries. The dried powder of G.lucidum was popular as a cancer chemotherapy agent in ancient China. G.lucidum clearly demonstrates anticancer activity in experiments with cancer cells and has possible therapeutic potential as a dietary supplement or alternative therapy for breast and prostate cancer. And also the fruiting bodies of Ganoderma lucidum (Polyporaceae) are a well known Chinese crude drug identified as a phytotoxic agent. However, since G.lucidum was available from different sources, it is advisable to test its biological activity.

Methodology: G.lucidum strains collected was isolated and identified with potato dextrose agar medium. Then the secondary metabolites were qualitatively studied through phytochemical analysis.

Outcome: Significant root length inhibition was observed at 100ppm and 200ppm. Similarly seed germination was also significantly inhibited at the concentration 100ppm and 200ppm extracts. The mean data of root length inhibition by ethanol extract in 100ppm was 0.393 and 0.208 in 200ppm. Likewise the mean data of seed germination inhibition by ethanol extract in 100ppm was 15 and 0.208 in 200ppm. Overall results supported that Ganoderma lucidum clearly supported the wider medicinal uses and established its anticancer activity.
\end{abstract}

Key words: Bracket fungus, Ganoderma lucidum, Phytochemicals, Radish seed, Seed Germination, Phytotoxicity.

\section{INTRODUCTION}

Medicinal mushrooms are mushroom used in the practice of medicine. Many species of mushrooms have been used in folk medicine for thousands of years. ${ }^{1}$ Medicinal mushrooms are now the subject of study for many ethno botanists and medical researchers. It is estimated that there are approximately 1.5 million species of mushrooms in the world of which 70,000 species are described. About 10,000 of the known species belongs to the macro fungi of which about 5000 species are edible and over 1,800 species are considered to have medicinal properties $^{2}$. 
G.lucidum has been used for promotion of vitality, longevity, prevention and treatment of various human diseases in China and other Asian countries. ${ }^{3}$ It was used for the treatment of asthma, diabetes, altitude sickness, cardiovascular disease, AIDS and Cancer. ${ }^{4,5}$ G.lucidum appears to be very safe since oral administration of the extracts does not display and toxicity ${ }^{6,7}$ and its merits have been investigated as a potential prophylactic agent for human health. ${ }^{8}$

G.lucidum and related species have the longest historical medicinal usage dating back at least four thousand year. In Japan it was called "keishi or mushroom of immortality". Traditionally it has been used widely in the treatment of hepatopathy. The ability of same mushrooms to inhibit tumor growth and enhance some aspects of the immune system has been a subject of research for approximately 50 years. ${ }^{9}$

Lingzhi was the name of G.lucidum and its close relative Ganoderma tsugae. G.lucidum enjoys special veneration in Asia, where it has been used as a medicinal mushroom in traditional Chinese medicine for more than 4000 years, making it one of the oldest mushrooms known to have been used in medicine. ${ }^{10}$

The word lingzhi, in Chinese, means "herb of spiritual potency" and has also been described as "mushroom of immortality". Because of its presumed health benefits and apparent absence of side-effects, it has attained a reputation in the East as the ultimate herbal substance. ${ }^{11}$

The mechanisms by which G.lucidum may affect cancer are unknown and they may target different stages of cancer development: through inhibition of angiogenesis (formation of new, tumor-induced blood vessels, created to supply nutrients to the tumor) mediated by cytokines, cytotoxicity, inhibiting migration of the cancer cells and metastasis, and inducing apoptosis of tumor cells. ${ }^{12}$

\section{MATERIALS AND METHODS}

\subsection{Collection}

Fruiting bodies of G.lucidum was collected from various places around Thanjavur, Thiruvarur and Nagapattinam districts. Then it was transported to laboratory using a clean poly ethylene bag.

\subsection{Isolation of G. lucidum ${ }^{13}$}

Tissue pieces of Ganoderma fructification were surface sterilized with tap water and $0.1 \%$ mercuric chloride solution, then rinsed for $2 \mathrm{~min}$ in distilled water. Fruiting bodies of G.lucidum isolates were inoculated onto potato dextrose agar (PDA) medium. After inoculation the fungal cultures were purified using pure culture technique and stock culture was maintained in PDA slants for further studies.

\subsection{Identification}

For confirmation of identification, taxonomic keys and descriptions were consulted ${ }^{14}$. Descriptions of basidiomycetes were made according to their macro, micro and cultural features by using standard manuals such as Manual of soil fungi ${ }^{15}$, Dematiaceous Hyphomycetes ${ }^{16}$. Colony color, morphology, hyphal structure, spore size and spore bearing structures were identified and compared. 


\subsection{Microscopical observation}

Morphological observations mainly followed by different methods. ${ }^{17}$ Lacto phenol cotton blue staining was used as the mounting medium. Microscopic characters were observed using a light microscope. For microscopic observation dermic elements were carefully examined and measured in thin sections perpendicular to the pileus surface.

\subsection{Grinding}

The sporocarps were cut in to small pieces, dried at $40^{\circ} \mathrm{C}$ for 48 hours and powdered. In each step, the plant material was dried to remove moisture and overcome the fungal contamination. The air-dried powder was stored in an air tight container for further use. ${ }^{18}$

\subsection{Extract preparation}

Various extracts of the experimental samples were prepared according to the methodology of Indian pharmacopoeia. ${ }^{19}$ The chemical nature and physical state of the mushroom powder make it difficult to dissolve in distilled water. So, the plant materials were soaked in distilled water for $24 \mathrm{hrs}$. One gram of powder was dissolved separately in $10 \mathrm{ml}$ ethanol, diethyl ether, chloroform and distilled water in cleaned screw cap bottle for $24 \mathrm{hrs}$. After $24 \mathrm{hrs}$ the dissolved extracts from the bottles were transferred to centrifugal tubes and centrifuged at 3000rpm for 10min. The centrifuged extracts (supernatant) were again re-centrifuged and filtered with Millipore filter. The filtered solvents with dissolved chemicals were concentrated and stored separately in refrigerated at $4^{\circ} \mathrm{C}$.

Qualitative screening of phytochemicals from mushroom extract ${ }^{20,21}$

\begin{tabular}{ccc}
\hline S.No & Phytochemicals & Chemicals added \\
\hline 1. & Tannins & $2 \mathrm{ml}$ ethanol extract $+3 \mathrm{ml} \mathrm{D} . \mathrm{H}_{2} \mathrm{O}+2 \mathrm{drops} 5 \% \mathrm{FeCl}_{3}$ \\
2. & Alkaloids & $200 \mathrm{mg}$ mushroom powder $+10 \mathrm{ml}$ methanol $+1 \% \mathrm{HCl}$ \\
3. & Saponins & $5 \mathrm{ml}$ extract $+5 \mathrm{ml} \mathrm{D} . \mathrm{H}_{2} \mathrm{O}$ \\
4. & Cardiac glycosides & $2 \mathrm{ml}$ filtrate $+1 \mathrm{ml}$ glacial acetic acid $+\mathrm{FeCl}_{3}+\mathrm{Con}_{2} \mathrm{H}_{2} \mathrm{SO}_{4}$ \\
5. & Terpenoids & $2 \mathrm{ml}$ filtrate $+2 \mathrm{ml}$ acetic anhydride $+\mathrm{Con}_{2} \mathrm{H}_{2} \mathrm{SO}_{4}$ \\
\hline
\end{tabular}

The extracts were subjected to various tests to screen phytochemicals tannins, alkaloids, saponins, cardiac glycosides and terpenoids.

\subsection{Radish seed phytotoxicity assay}

To evaluate phytotoxic properties of extract of Ganoderma lucidum, radish seed phytotoxicity assay were done for this purpose. ${ }^{22}$

\subsection{For root length determination}

Whatman No: 1 filter paper kept on petridish and 5ml extracts (100ppm and 200ppm) were added separately. Filter paper was dried at room temperature for reducing extra solvent. $5 \mathrm{ml}$ DDW was added and then 20 radish seeds were placed on petri dishes followed by tightly sealed and incubated at $23 \pm 2^{\circ} \mathrm{C}$. Root length was measured after 1,3 and 5 days of interval. Only DDW containing petridish was used as control. Each assay was carried out in three times. 


\subsection{For seed germination determination}

This part of the determination was similar to that of earlier determination. Here also two concentration that (100ppm and 200ppm) were used. Germinated seeds were counted after every day up to 5 days. Each experiment was carried out in three times.

\section{RESULTS AND DISCUSSION}

Table: 1 collection of Ganoderma lucidum from various places.

\begin{tabular}{cccc}
\hline S.No & Location & Substrate & Strain no \\
\hline 1 & Paeraiyur & Bambusa vulgaris & VAMNG-I \\
2 & Mulaiyur & Bambusa vulgaris & VAMNG-II \\
3 & Kudikadu & Red Soil & VAMNG-III \\
4 & Cholapuram & Morinda oleifera & VAMNG-IV \\
5 & Aadudhurai & Cocos nucifera & VAMNG-V \\
6 & Pandhanallur & Syzygium cumini & VAMNG-VI \\
7 & Sirkali & Musa paradisiacal & VAMNG-VII \\
8 & Ammachatthiram & Barks & VAMNG-VIII \\
9 & Valangaiman & Barks & VAMNG-IX \\
10 & Sundhara Perumal Kovil & Alluvial Soil & VAMNG-X \\
\hline
\end{tabular}

\subsection{Microscopical structure of spores and hyphae of G.lucidum}

Basidiomata annual, stipate brilliantly laccate, mycelia mat white to light cream scattered, more or less extensive, much branched thin walled hyphae often described as "witches broom". Aerial mycelium and the lateral branches may become very short. Thick walled hyaline, branched aseptate 1.5-3.0mm, Ellipsoid to avoid, 8.5-12.5 $\times 5.5-7.5 \mathrm{~mm}$. In these findings the microscopical structure of mycelium was aerial and has thick walled hyaline.

\subsection{Morphological identification}

The structure of the pileal crust and cortex are usual characters in the taxonomy of Ganodermataceae. The former character occurs mainly in Ganoderma and Amauroderma. Fruitbodies of Ganoderma mostly have hymenioderm, and anamixoderm. ${ }^{23}$ For species identification, however, hyphal characters are often useful. ${ }^{24}$ The naturally produced basidiocarps of G.lucidum shows various morphological characteristics; sessile, stipitate, imbricate, non- imbricate.
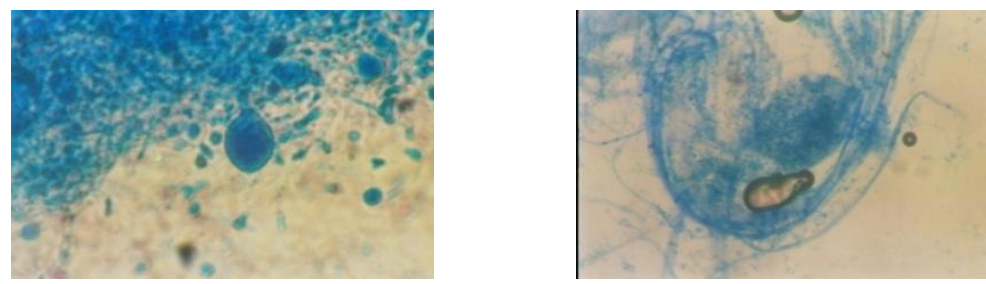

Fig: 1.Spores and hyphae Of G.lucidum 


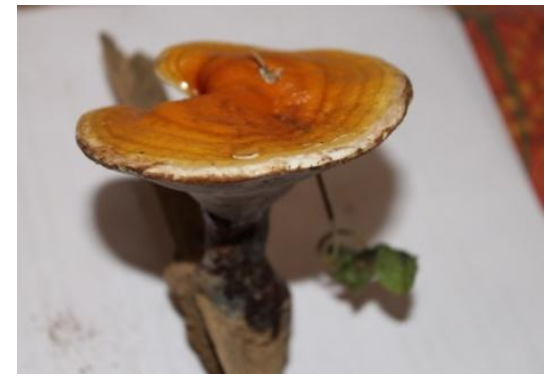

Fig: 2. Fruiting body of G.lucidum

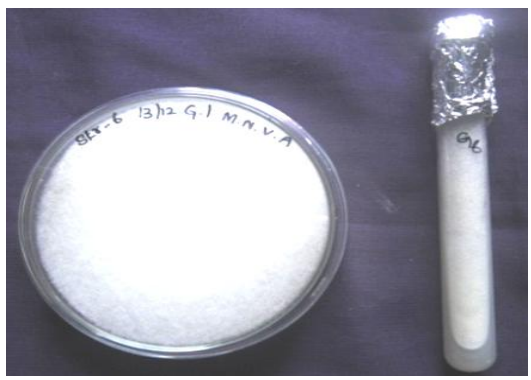

Fig: 3. Pure culture of G.lucidum

G.lucidum was a basidiomycete's mushroom with pores underneath the cap. The basidiocarps was dark red to brown with a lacquered appearance but often cream or yellowish towards the margin, corky or woody and circular to semicircular to fan shaped. The stalk was dark brown to black, hard and eccentric, but sometimes the stalk was absent. The hymenophores compose of tiny circular pores, white and smooth surface. The spore was ovoid with double layer.

\subsection{Cultural identification}

Growth rate moderate to rapid 1.5-3.5/week, covering petridish in 12-15 days Advancing zone white hyaline even appressed. Mycelial mat white to light cream, scaltered, more or less extensive. Hyphae in the advancing zone hyaline, thin walled. Texture of mycelia mat appressed farinaceous felty.

Table: 2 Phyto chemical analyses on G.lucidum extract

\begin{tabular}{cccc}
\hline S.No & Phytochemicals & Observation & Inference \\
\hline 1. & Tannins & Blue black precipitate & $(+)$ \\
2. & Alkaloids & Brownish red precipitate & $(+)$ \\
3. & Saponins & Froth formation & $(-)$ \\
4. & Cardiac glycosides & Green blue precipitate & $(+)$ \\
5. & Terpenoids & Blue green ring & $(+)$ \\
\hline
\end{tabular}

(+) Indicates presence of phytochemicals (-) Indicates absence of phytochemicals

Preliminary phytochemical screening of G.lucidum unprocessed powder, reveals the presence of phytochemicals which are absent in the fractionated extracts, this was a possible explanation to metabolites formation in the body when extract of this mushroom was ingested and formed metabolites may be responsible for the bioactive properties of this mushroom. ${ }^{26}$ In these study alkaloids, cardiac glycosides, tannins, terpenoids shows positive results and saponin shows negative results. The presence of alkaloids in the mushroom powder explains anti-bacterial activity; since this phytochemical was reported to have anti-bacterial activity. The presence of tannins which can complex with the metal ions and macromolecules such as proteins and carbohydrates obtained in the powdered sample can be utilized in weight reduction management. ${ }^{27}$ Mushroom extracts of G.lucidum were prepared and phytochemical analysis under taken using various methods to identify the constituents of the mushroom ${ }^{28}$. 
Triterpenoids are the bitter tasting phytochemical which gives the extract its bitter taste, the terpenoids are said to form complexes with steroids to provide the said anti- inflammatory effects of this wild mushroom and equally, its anti-bacterial activity. Presence of these phytochemical elements indicates that Indian medicinal mushrooms like G.lucidum are potential sources of anti-tumor activity. G.lucidum possess profound antioxidant and anti tumor activity. In these study alkaloids, cardiac glycosides, tannins, terpenoids shows positive results and saponin shows negative results.

\subsection{Radish seed Phytotoxicity assay \& Seed germination}

Table 1, 2 and Fig 1, 2 shows the inhibitory effect of G.lucidum against radish seeds. Statistical analysis proved that root length was significantly inhibited by the extracts at both the concentrations 100ppm and 200ppm.

In another cases, seed germinations were also significantly inhibited by the both concentrations $100 \mathrm{ppm}$ and 200ppm. These results were analyzed compared with control. These observations may be accomplished due to the presence of active biological compounds.

Table: 3. Analysis of mean data of root length inhibition by ethanol extract of Ganoderma lucidum

\begin{tabular}{|c|c|c|c|c|c|}
\hline Variables\& concentration & root length $(\mathrm{mm})$ & Days & Day 1 & Day 3 & Day 5 \\
\hline Control & 14.315 & Control & 8.56 & 13.63 & 20.75 \\
\hline 100ppm & 0.393 & 100ppm & 0.25 & 0.37 & 0.59 \\
\hline 200ppm & 0.208 & 200ppm & 0.08 & 0.21 & 0.33 \\
\hline
\end{tabular}

Table: 4 Analysis of mean data of Seed Germination, inhibition by ethanol extract of G. lucidum

\begin{tabular}{ll}
\hline Variables \& Concentration & \%Seed Germination \\
\hline Control & 85 \\
$100 \mathrm{ppm}$ & 15 \\
$200 \mathrm{ppm}$ & 0.208
\end{tabular}

\begin{tabular}{cccc}
\hline Days & Control & 100ppm & 200ppm \\
\hline Day 1 & 7 & 0 & 0 \\
Day 2 & 9 & 0 & 0 \\
Day 3 & 12 & 1 & 0 \\
Day 4 & 14 & 2 & 1 \\
Day 5 & 17 & 3 & 2 \\
\hline
\end{tabular}

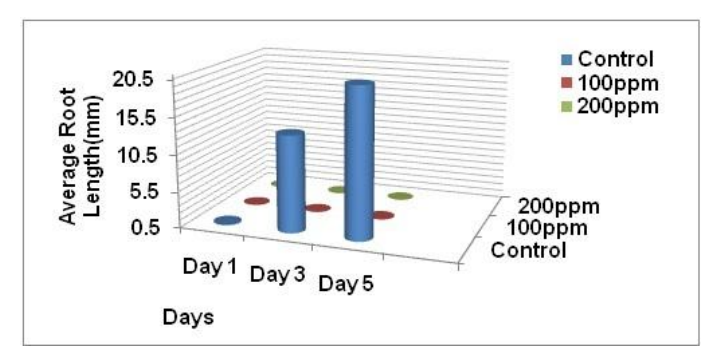

Fig.3

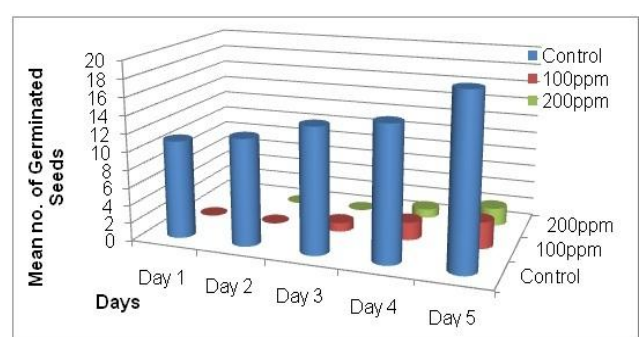

Fig.4

Fig: 3. Histogram shows regular root length inhibition by the ethanol Extract at two different concentrations (100ppm and 200ppm) of G. lucidum. Data compared with control.

Fig: 4. Histogram shows phytotoxicity assay on radish seed germination percentage at two different concentrations (100ppm and 200ppm) of G. lucidum. Data compared with control. 


\section{CONCLUSION}

Since 1970s numerous mushroom fungi have been increasingly used as a source of medicinal compounds and therapeutic adjuvants or health food supplements. It contains phytpochemical compounds and also the phytotoxicity assay may be accomplished due to the presence of active biological compounds. In drug discovery, the major secondary metabolites are of potential medicinal interest.

Drug discovery is the key attempt of our age to overcome many life-threatening diseases like cancer. Plant-based compounds have been playing an important role in the development of several clinically useful anticancer agents i.e., including taxol, vinblastine, vincristine, the camptothecin derivatives, topotecan and irinotecan and etoposide derived from epipodophyllotoxin. ${ }^{29}$

Different studies have shown many secondary metabolites as a source of bioactive compounds with allelo chemical potential have great chemical diversity and are involved in many metabolic and ecological processes. ${ }^{30}$

In drug discovery, the major secondary metabolites (terpenoids, phenolics and alkaloids) are of potential medicinal interest. The mentioned structure diversity is reflected in a variety of biological activities as, for instance, inhibitors of enzymes and antitumor, immunosuppressive and anti parasitic agents. ${ }^{31}$

These findings may be attributed to the nature of biological active compounds and their strong solubility with appropriate solvent. The clinical study indicated that G.lucidum was well tolerated and could improve the immunity. However, sometimes it is often better to use alcohols or hydro alcoholic solutions after partial lipid removal ${ }^{32}$. Many researchers have already been used ethanol as a solvent for evaluating cytotoxicity, phytotoxicity, antibacterial, antitumor activity in several plant species. ${ }^{33}, 34$ However, intensive and extensive investigations are needed to establish their therapeutic potential. Further study is required for isolating specific compound.

Ganoderma lucidum is the valuable medicinal mushroom for its multipurpose uses. The present study has been undertaken to identify this plant as a source of phytotoxic agent for giving a basic platform for anti cancer studies. Specifically ethanol extract of G.lucidum has been studied using different bioassay for this purpose ${ }^{35}$.

The experimental findings reveal that G.lucidum occurring in South India has potential effect for anticancer activity. At present we are collaborating with another laboratory to study the potential of Ganoderma lucidum extract in complimentary cancer therapy through a clinical study of cell lines and Wister albino rats analysis.

\section{ACKNOWLEDGEMENT}

The authors are thankful to the Secretary and Correspondent, A.V.V.M.Sri Pushpam College, Poondi, Thanjavur for providing laboratory facilities and Department of Science and Technology for granting financial assistance to "INSPIRE FELLOW" for research work. 


\section{REFERENCES}

1. Christopher Hobbs. Medicinal mushrooms, An Exploration of Tradition Healing and Culture, Herbs and Health Series, 1998; I (1): 27-33.

2. Chang, S.T. Ganoderma The leader in production and technology of mushroom nutriceuticals. In Proc 6th Int symp. Recent Adv. The pharmaceutical Society of Korea, Seoul, Korea.1995, pp 43-52.

3. Lu, Q. Y., Jin, Y. S., Zhang, Q., Zhang, Z., Heber, D., Go, V. L. W. Ganoderma lucidum extracts inhibit growth and induce actin polymerization in bladder cancer cells in vitro. Cancer Letters 2004; 216: 9-20.

4. Shiao, M. S. Natural products of the medicinal fungus Ganoderma lucidum: Occurrence, biological activities, and pharmacological functions. The Chemical Record 2003; 3: 172-180.

5. Wasser, S. P and Weis, A. L. Medicinal properties of substances occurring in higher basidiomycetes mushrooms. International Journal of Medicinal Mushrooms 1999; 1: 31-62.

6. Eo, S. K., Kim, Y. S., Lee, C. K and Han, S. S. Antiviral activities of various water and methanol soluble substances isolated from Ganoderma lucidum. Journal of Ethnopharmacology 1999 ;(a) 68:129-136.

Eo, S. K., Kim, Y. S., Lee, C. K and Han, S. S. Anti therpetic activities of various proteins bound polysaccharides isolated from Ganoderma lucidum. Journal of Ethnopharmacology 1999 ; ( b) 68:175-181.

7. Kim, K. C \& Kim, I. G. Ganoderma lucidum extract protects DNA from strand breakage caused by hydroxyl radical and UV irradiation. International Journal of Molecular Medicine 1999; 4: 273-277.

8. Lindequist, U. Niedermeyer, T.H.J. and Julich, W.D. The pharmacological potential mushroom. Evidence based complementary and alternative medicine 2005; 2(3): 285-299.

9. Benny K H. Immunomodulatory and Antimicrobial Effects of Traditional Chinese Medicinal, Department of Pharmacology, Faculty of Medicine, 1999, 5(2): 85-88.

10. Dan Bensky. Chinese Herbal Medicine, Materia Medica India 2004; 7(5): 125-128.

11. Mueller C I, Kumagai T O, Kelly J, Seeram N P, Heber D K, Koeffler H P. Ganoderma causes apoptosis and multiple myeloma Leukemia Research 2006; 30(7): 841-848.

12. Chen, A.W. and W.W.L. Hu. Strategies for obtaining monocaryons in Ganoderma species. Ganoderma: Systematics, Sathology and Sharma-cology proceedings of contributed symposium. 59 A,B, $5^{\text {th }}$ International Mycological Congress, Vancouver.1993.

13. Ryvarden, L. Genera of Polypores. Nomenclature and taxonomy. In Synopsis Fungorum 5. Fungiflora, Oslo, Norwa. 1991, 2, pp. 363.

14. Gillman J.C., Sexual spores, A manual of soil fungi. $2^{\text {nd }} E d, 1957$, Pp 4.

15. Ellis, M.B. Dematiaceous Hyphomycetes. The Commonwealth Mycological Institute, England. 1971, pp.1-608.

16. Wang, $\mathrm{H}$ and Ng, T.B. "Ganodermin", an anti-fungal protein from fruiting bodies of the mushroom, Ganoderma lucidum. Peptides, 2006, 27 (1): 27-30.

17. Dandan Liua, Zheng Hua, Zhiang Liua, Bo Yanga, Wenjuan Tub and Liang Lia. Chemical composition and antimicrobial activity of essential oil isolated from the cultured mycelia of Ganoderma japonicum, Laboratory Industrial Microbiol, 2009; 9(3): 122-125.

18. Anonymous Shen-Nong Materia Medica. Beijing: People's Hygiene Press. (102-200AD, reprinted in 1955) Pp514.

19. Allen, S.T. Chemical analysis of ecological material, Blackwell Scientific Publication, New York.1976, Pp 313.

20. Harborne, J. B. Phytochemical methods, First Edition, Chapman and Hall Ltd, London. 1976, 52 -55.

21. Turker, A.U. and N.D. Camper. Biological activity of common mullein, a medicinal plant. J. Ethnopharmacol 2002; 82: $117-125$.

22. Steyaert, R.L. Study of some Ganoderma species. Bulletin du Jardin Botanique National de Belgique, 1980; 50: 135-186.

23. Zhao J.D. The Ganodermataceae in China. Bibliotheca Mycologica. J.Cramer Berlin Stuttgart, 1989, Pp 132.

24. Shin, G.C. and Geo, G.S. Formation of non-basidiocarpous basidiospore of Ganoderma lucidum. Korean Journal of Mycology 1988; 16:230-234.

25. Idowu, Y.O. Onawunmi, G.O. Ogundani, A.O and Adesanya, S.A. Antimicrobial constituents of Crysophyllum albidum seed: Cotyledon. Nigerian Journal of veterinary production and medicine. 2003; 7:33-36.

26. Dei, H.K. Rose, S.P and Mackenzie A. Shea nut (Vitellaria paradoxa) meal as a feed ingredient for poultry. In World Poultry Science Journal. 2007; 63(4): 611-624.

27. Smith, B.J. \& Sivasithamparam, K. Morphological studies of Ganoderma (Ganodermmataceae) from the Australasian Pacific regions. AustralianSystematic Botany. 2003; 16: 487-503.

28. Shoeb, M. Anticancer agents from medicinal plants. Bangladesh J. Pharmacol 2006; 1: 35-41.

29. Einhellig, F.A., Allelopathy - a natural protection, allelochemicals. In: Handbook of natural pesticides: methods v.1 theory, pratice and detection. Ed., Mandava N.B. CRC Press LLC, 1985, Pp: 161-200.

30. Carmichael J, DeGraff WG, Gazdar AF, Minna JD, Mitchell JB. Evaluation of a tetrazolium-based semiautomated colorimetric assay: assessment of chemosensitivity testing. Cancer Res 1987; 47: 936-42

31. Marston, A. and K. Hostettmann. Plant Plenum Press. saponins: chemistry and molluscicidal action. In: 45. Karpas, A., 1982. Viruses and leukemias. Terpenoids. Eds., American Ecological Chemistry and Biochemistry of Plant Scientist 1991; 70: $277-285$ 
32. Hussain, A., M. Zia and B. Mirza. Cytotoxic and antitumor potential of Fagonia cretica L. Turkish J. Biol. 2007; 31: 19-24.

33. Inayatullah, S., R. Irum, M. Ateeq-ur-Rehman, for plant screening and fractionation. In: Methods in F. Chaudhury and B. Mirza. Biological Plant Biochemistry. Ed., Hostettmann K. Vol 6. Evaluation of some selected plant species of Pakistan .Academic Press, pp: 1-31. Pharmaceutical Biol. 2007; 45(5): 397-407.

34. Hobbs C. Medicinal Mushrooms: An exploration of tradition, healing and culture. Santa Cruz, CA: Botanica Press; 1995; 96-107. 